\title{
El dolor y el sufrimiento como claves hermenéuticas para la comprensión de las concepciones del mundo y de la vida*
}

\author{
Pain and suffering as hermeneutical keys \\ for the understanding of Weltanschauungen
}

\author{
FRANCISCO JAVIER CORTÉS SÁNCHEZ \\ Universidad de Salamanca
}

Recibido: 02-11-2018 Aceptado: 17-11-2018

\begin{abstract}
RESUMEN:
El hecho de que las variables socioculturales influyan en la interpretación del dolor y el sufrimiento conducen a replantear el modo de comprender a los seres humanos. En el presente artículo se reflexiona acerca del dolor y el sufrimiento desde su dimensión simbólica. De este modo, se presenta las Weltanschauungen como clave de comprensión e interpretación del ser humano tomando como punto de partida el dolor y el sufrimiento.
\end{abstract}

\section{PALABRAS CLAVE:}

WELTANSCHAUUNG, DOLOR, SUFRIMIENTO, INTERSUBJETIVIDAD

\footnotetext{
* El presente trabajo es fruto de dos conferencias. La primera en el evento: Primer Seminario de Investigación USAL-IB3 Filosofía del dolor, celebrado en la Universidad de Salamanca en los días 15/12/2016 y 16/12/2016 y organizado por el Proyecto de Investigación USAL-IB3 Filosofía del dolor. La segunda en el evento: International Congress / Congreso Internacional. Philosophy of pain / Filosofía del dolor, celebrado en la misma universidad en los días del 02/05/2018 al 04/05/2018.
}

(C) Contrastes. Revista Internacional de Filosofia, vol. XXIII-No3 (2018), pp. 11-25. ISSN: 1136-4076 Departamento de Filosofía, Universidad de Málaga, Facultad de Filosofía y Letras Campus de Teatinos, E-29071 Málaga (España) 
ABSTRACT:

The sociocultural variables influence the interpretation of pain and suffering. This fact leads to rethinking the way of understanding humans. In this article we reflect on pain and suffering from its symbolic dimension. In this way, Weltanschauungen is presented as a key to understanding and interpreting humans, taking pain and suffering as a starting point.

KEYWORDS:

WELTANSCHAUUNG, PAIN, SUFFERING, INTERSUBTECTIVITY

\section{Cuestiones PRELIMINARES ACERCA DEL DOLOR}

TRADICIONALMENTE SE HA CONSIDERADO que el acceso a la reflexión y, más concretamente, al filosofar, deviene de la admiración y el asombro. Esta consideración, se instituye desde que Platón dijera en el Teeteto que Iris (representación de la ciencia y la sabiduría) era hija de Taumas (representación del asombro). Idea en la que insiste Aristóteles en su Metafísica: las ciencias tienen su origen en la admiración o el asombro. $\mathrm{Y}$ es que, sin lugar a duda, el problema del acceso a la reflexión y a la propia filosofía, es una cuestión filosófica que está en íntima conexión con la concepción del ser humano. Pero la idea del asombro como acceso, contiene una interpretación intelectualista de lo que es el origen y el deseo del conocimiento. El filósofo español Carlos París, en cambio, afirma que el origen del filosofar no se reduce a un deseo intelectual, sino que también contiene una relación ética con los otros. El grito, el llanto, el lamento, por tanto, también son parte del acceso al filosofar por iniciar una búsqueda de la justicia. ${ }^{1}$

El grito, el llanto y el lamento son expresiones de dolor y sufrimiento. Para Aristóteles el dolor era considerado como una forma peculiar de la emoción; posteriormente, para Descartes — desde su dualismo ontológico - el dolor es una sensación producida en el mecanismo corporal. ${ }^{2}$ Siguiendo esta estela, las ciencias naturales han estudiado el dolor como experiencia sensorial fisiológica. De este modo, cuando pensamos en el dolor, solemos pensar en el dolor somático, es decir, lo relativo al cuerpo doliente. Afecciones tales como pueden ser un simple catarro o una jaqueca, o el hecho de recibir un golpe, todas ellas son experiencias subjetivas y relativas al soma, al cuerpo. Pero, también se medita acerca del dolor en su acepción psicológica y, en este sentido, se amplía la concepción a través del término sufrimiento. Ejemplos de ello son la angustia, la ansiedad, la depresión, etc. No obstante, ambas acepciones siguen apelando a la subjetividad sentiente. Ambas experiencias mencionadas, que separamos de

1 Cf. París 2014, p. 21

$2 C f$. Le Breton 1999, p. 10 
manera analítica en somática (cuerpo) y psicológica (mente), conforman un ente unitario - un ser psicofísico en terminología diltheyana-, y son universales. ${ }^{3}$

La experiencia del dolor y del sufrimiento es vivida por el conjunto de la especie humana y por otras especies sentientes (si bien se conocen excepciones individuales como el caso de quienes padecen enfermedades como el síndrome de Riley-Day, por ejemplo). Si bien la experiencia del dolor es privativa — dado que el doliente tiene vivencia de su dolor en primera persona-, no se reduce a la dimensión subjetiva. El dolor tiene una dimensión social de intersubjetividad que lleva a Le Breton a afirmar:

Por medio de la compasión o la culpabilidad que siempre induce en el otro, el dolor es un medio seguro para estar acompañado, compadecido, amado, y sobre todo reconocido en el estatuto particular que el mismo dolor ofrece. ${ }^{4}$

La queja o el llanto, como expresiones de la experiencia dolorosa y sufriente no solo son respuestas subjetivas algopáthicas ${ }^{5}$ sino que también son fuente de reclamos de la atención y el afecto del Otro. La compasión, la condolencia, la simpatía, la empatía, remiten incluso en su etimología a esta dimensión intersubjetiva del dolor. A la dimensión subjetiva del dolor y el sufrimiento, y a la dimensión intersubjetiva de los mismos, se suma la dimensión simbólica, que afecta tanto a la vivencia privada como a la relación para con los demás. Desde las investigaciones de Mark Zborowski, pasando por los estudios de Irving Kenneth Zola y los de C. Koopman, S. Eisenthal y J.-D. Stoeckle, ${ }^{6}$ se puede sostener que las vivencias del dolor dependen del contexto, la situación personal así como de la cultura. Todo ello hace replantearnos la concepción acerca del ser humano.

De este modo, en este artículo se presenta una comprensión del dolor y el sufrimiento desde una perspectiva filosófica, entendiendo la filosofía como saber de segundo grado, ${ }^{7}$ un saber y actividad crítica implantada e inmersa en el presente práctico. Esta consideración conlleva a su vez y de manera inexorable, una concepción antropológica que considera a los seres humanos como seres simbólicos.

La postura que se expone en este artículo no tiene que ver con la concepción de la filosofía como filosofía primera donde, establecida de manera a priori un esquema general, posteriormente se aplicara este al mundo. No se parten

3 Cf. Serrano de Haro 2012, p. 16

4 Le Breton 1999, p. 231

5 Se utiliza el término algopáthico para hacer referencia al dolor (algos) padecido (pathos).

6 Cf. Zborowski 1952, pp. 16-30; Zola 1966, pp. 624-625; Koopman, Eisenthla y Stoeckle 1984, pp. 487-490. Referencias sacadas de Le Breton 1999, pp. 148-158

7 Para una definición de la filosofía como saber de segundo grado consultar: Bueno 1995. 
de axiomas o fundamentos, sino más bien el proceso es inverso. Se toma la filosofía como un saber de segundo grado cuya tarea consiste en ofrecer una explicación holística a partir de esas ciencias y saberes particulares. Se considera que dichos estudios son investigaciones que se trazan en acotaciones ónticas concretas, pero no piensan los fenómenos en su totalidad, tarea que sí asume la filosofía. Para comprender esto, podemos pensar en el fenómeno del dolor desde la acotación óntica del cuerpo vivo estudiado desde la fisiología, donde el dolor es explicado como un proceso de estímulos bioquímicos. Que esto sea cierto, no niega que la experiencia subjetiva del dolor sea considerada, por ejemplo, como insoportable - que haría referencia a la unidad psicosomática-. Pero este mismo fenómeno, también puede ser interpretado como dolor merecido, lo que estaría haciendo referencia a su acotación óntica de lo simbólico.

Si los estudios relativos al dolor que han manejado variables culturales concluyen que los aspectos socioculturales influyen en las experiencias algopháticas, cabe replantearse la concepción antropológica que posibilite comprender dicho fenómeno. La pregunta que subyace de los estudios relativos al dolor que introducen variables culturales es la siguiente: ¿cómo entender la pluralidad de sentidos del dolor?

\section{CONCEPCIONES DEL MUNDO Y DE LA VIDA.}

EN TORNO AL DOLOR Y EL SUFRIMIENTO PADECIDO

Todos los humanos necesitamos tener una especie de brújula vital que nos permita orientarnos en el mundo y en la vida. Además de brújula, necesitamos un mapa que nos permita situarnos. La situación y la orientación confieren sentido a nuestro existir, nos ofrece certezas y seguridades. Este mapa y brújula vital es lo que Dilthey denominaba como Weltanschaunng. Este concepto ha sido traducido en ocasiones como cosmovisión. Julián Marías lo tradujo como «visión del mundo». ${ }^{8}$ Pero parece más correcto utilizar «concepción del mundo» tal y como hizo Eugenio Imaz, traductor al castellano de las obras completas de Dilthey, dado que las Weltanschauungen no son meras representaciones, imágenes o visiones del mundo, sino que son modos de ser y existir, es decir: son modos de vivir. Es por ello que el propio Dilthey utilizara el término Welt-und-Lebensansicht para hacer referencia a las visiones del mundo y de la vida - que conformarían una de las partes de las concepciones del mundo-, mientras que usó el término Lebens-und-Weltanschauung para hacer referencia a las concepciones del mundo y de la vida. Dilthey expresa así esta idea:

Todas las concepciones del mundo contienen, cuando tratan de ofrecer una solución completa al enigma de la vida, la misma estructura. Esta estructura consiste 
siempre en una conexión en la cual se decide acerca del significado y sentido del mundo sobre la base de una imagen de él, y se deduce así el ideal, el bien sumo, los principios supremos de la conducta. ${ }^{9}$

De esta manera, toda concepción del mundo y de la vida confiere sentido y significado a nuestra propia existencia desde una idea del mundo y de la vida que no se reduce a una imagen de las mismas, sino que contiene estimaciones y valoraciones. Es por ello que Dilthey comenta que en el ser psicofísico se da una estructura triple indisoluble de representación, volición y afecto sobre el mundo y la vida. ${ }^{10}$ Las concepciones del mundo y de la vida, por tanto, suponen tanto un modo de comprender el mundo y la vida, como un modo de valorarlos. Aunque el antropólogo Clifford Geertz diferencia entre ethos y cosmovisión, se puede decir que las concepciones del mundo y de la vida son el conjunto de esa división analítica entre el ethos y la cosmovisión tal y como se encuentra en la siguiente definición:

El ethos de un pueblo es el tono, el carácter y la calidad de su vida, su estilo moral y estético, la disposición de su ánimo; se trata de la actitud subyacente que un pueblo tiene ante sí mismo y ante el mundo que la vida refleja. Su cosmovisión es su retrato de la manera en que las cosas son en su pura efectividad; es su concepción de la naturaleza, de la persona, de la sociedad. La cosmovisión contiene las ideas más generales de orden de ese pueblo. ${ }^{11}$

Toda persona tiene una concepción del mundo y de la vida y, aunque propia, siempre es compartida. Su configuración no es individual sino que surge del entramado social y se va adquiriendo una vez venimos al mundo y nos vamos haciendo a él. De este modo, la asunción de la concepción del mundo y de la vida se convierte en un horizonte de sentido vital socialmente compartido. Las Weltanschauungen es lo que los antropólogos denominan como cultura. Lo que une a las religiones, los mitos y la filosofía, es que todas ellas intentan descifrar el enigma de la existencia y otorgan, a su vez, sentido a la misma. Nos encontramos con una expresión similar — salvando las diferencias particulares que se encuentran dadas en cada formulación - cuando Husserl define el mundo de la vida (Lebenswelt) de la siguiente manera:

El mundo de la vida [...] está siempre ya ahí, siendo para nosotros de antemano el «suelo» para todos, se trate teorética o extra-teorética. El mundo es para nosotros [...] pre-dado como horizonte no una vez accidentalmente sino siempre y

9 Dilthey 1978 , p. 115

10 Cf. Dilthey 1944

11 Geertz 2003, p. 118 
necesariamente como campo universal de una práctica efectiva y posible. Vivir es permanentemente vivir-en-la-certeza-de-mundo. ${ }^{12}$

No obstante, no se quiere interpretar las concepciones del mundo y de la vida de una manera idealista. Es decir, no se trata de una pluralidad de sentidos en los cuales, los seres humanos situados, estemos sumergidos de tal forma que no existan vasos comunicantes entre aquello que no es concepción del mundo así como con otras concepciones del mundo.

Las concepciones del mundo y de la vida, como mapamundis que son, suponen la idea de mundo. Si bien el mundo no es la totalidad de las cosas en el sentido de omnitudo rerum, el mundo sí es la totalidad de las cosas en tanto que son accesibles al radio de acción de nuestra praxis en la conformación de las mismas. ${ }^{13}$ De este modo, las concepciones del mundo suponen expresiones del estado del mundo desde el punto de vista antrópico. Para ilustrar esto nos valemos del siguiente ejemplo: Tomemos una comunidad A que interpreta el rayo como un acto fruto de la acción de los dioses; y una comunidad $\mathrm{B}$ que interpreta el rayo como una manifestación eléctrica producto de un proceso meteorológico. Ambas comunidades perciben el mismo fenómeno y, sin embargo, la interpretación del mismo difiere. La concepción del mundo y de la vida de la comunidad $\mathrm{A}$, se ha denominado tradicionalmente como mitológica, mientras que la concepción del mundo de la comunidad $\mathrm{B}$, se conoce como científica. De este modo, la interpretación del fenómeno no procede tan solo del acto perceptivo sino que se sustenta en un horizonte de sentido que es socialmente compartido y, en el caso de la comunidad A, el sustento procede de la cartografía mitológica, mientras que en la comunidad B proviene del sustento de la ceraunología. Lo importante de este ejemplo, aparte de exponer diferentes modos de concepción del mundo, es que nos conduce a la pregunta de cómo opera y es posible esta pluralidad de concepciones.

Para Dilthey el surgimiento de las concepciones del mundo y de la vida tiene que ver, principalmente, con la experiencia de la muerte de alguien que nos induce a pensar en la finitud de nuestra existencia. De este modo, con la finalidad de encontrar sentido a la finitud se le otorga un sentido a dicho acontecimiento. Y esto sería el nexo común entre las religiones y la filosofía.

Para Dilthey, la experiencia de la muerte de alguien conocido sería una vivencia dolorosa dado que nos muestra la finitud de nuestra existencia. Ante la pérdida de un ser querido las religiones, los mitos y la filosofía, generan relatos con el fin de encontrar algún sentido así como alguna explicación. Denomina a esto el enigma de la vida y de la existencia. Este enigma, según Dilthey, se nos

12 Husserl 2008, p. 184

13 Cf. Bueno 1995, p. 9 
presentaría de modo constante a lo largo de la historia y se presentaría a cada persona del mismo modo. Y así, la finitud de nuestra existencia nos conduce, según Dilthey, a preguntarnos qué es el mundo y qué debo hacer con mi vida.

El enigma de la existencia nos mira en todas las épocas con el mismo rostro misterioso, percibimos bien sus rasgos pero quisiéramos adivinar el alma que tras ellos se oculta. En este enigma se encuentran siempre radicalmente entrelazados el misterio de qué sea el mundo y la cuestión de qué es lo que yo tengo que hacer en él, para qué estoy en él, cuál ha de ser mi fin. ${ }^{14}$

No obstante, cada venida al mundo de cada uno de nosotros supone la existencia previa de una concepción del mundo en la cual crecemos y nos socializamos. De este modo, no se trata tanto de la cuestión de cómo surgen y si existe un único «principio» que de explicación a esta pluralidad de concepciones del mundo y de la vida, sino más bien el por qué, dada una misma naturaleza, existe la posibilidad de una pluralidad de concepciones del mundo y de la vida. Es decir, partimos de la facticidad de la pluralidad de concepciones del mundo y de la vida, lo que cabe reconstruir es la acepción antropológica que dé cuenta de esta misma facticidad.

Lo que nos sugiere la pluralidad de concepciones del mundo y de la vida es que el ser humano es un ser poiético en tanto que genera y dona sentido a su entorno. Pero es más, es un ser autopoiético en tanto que la idea que conforma de sí también lo transforma. De este modo lo entiende Nicol:

Cambia el hombre cuando cambian sus productos; cambian los productos porque cambia el productor [...] El creador de la historia es un ser que se crea a sí mismo históricamente. ${ }^{15}$

Esta concepción antropológica de la actividad performativa que construye sentido tanto de nosotros mismos como de aquello que no somos nosotros, explica la variedad de estilos y formas de vida. En otro pasaje Nicol comenta lo siguiente:

El hombre forma y reforma y transforma su individualidad históricamente [...] Las formas históricas del ser del hombre, las grandes etapas de sus cambios reales, son modos diferentes de integración en la comunidad. Esta integración, que como tal es un hecho permanente - el hombre no está solo nunca-, varía en cualidades, en direcciones, dijéramos también en intensidades. Estas variaciones 
determinan diferentes estilos de vida: estilos políticos y religiosos, económicos y artísticos, jurídicos, científicos y filosóficos. ${ }^{16}$

La idea que tengamos acerca del mundo y de la vida, la cual también nos incluye a los seres humanos que la conformamos, actúa de manera performativa en nuestras propias existencias. Es decir, las concepciones del mundo y de la vida son productos humanos pero, a su vez, los seres humanos somos resultado de dichas concepciones. Y estas concepciones están en relación estrecha con el entorno que generamos, desde las técnicas y tecnologías fabricadas, los espacios construidos, etc.

A modo de ejemplo ponemos el libro de Job del Antiguo Testamento. En este libro podemos ver cómo el dolor y el sufrimiento padecido por Job se comprende desde una teodicea en la cual el dolor sufrido adquiere sentido trascendental y organiza y estructura su existencia que se materializa en la persona de Job. La cuestión que subyace de la experiencia vivida por Job es la siguiente: ¿por qué ha de sufrir el justo? Pedro Fernández Liria lo plantea en los siguientes términos:

[C]uando la «lamentación» se convierte en «queja» o «protesta», esto es, cuando ya no preguntamos simplemente por qué hay mal, sino que preguntamos por qué he de sufrirlo precisamente yo y no otros, ya que no todos lo sufren [...] se pide una explicación que de cuenta de una diferencia o asimetría en la distribución del sufrimiento. Y la primera y más persistente explicación ofrecida a este respecto por la «sabiduría» es la de la retribución: «todo sufrimiento es merecido porque es el castigo de un pecado individual o colectivo, conocido o desconocido». Ello nos sitúa, como señala Ricoeur haciendo uso de la conocida expresión de Hegel, en la primera «visión moral del mundo». Pero, cuando, como tantas veces vemos, el dolor y el sufrimiento sin límite se ceba con el más pequeño y más débil, con el inocente, con el más justo... ¿no se vuelve la ley de la retribución contra Dios mismo? ¿No ha de ser recriminado Dios, en virtud de la misma ley por la que se justifica el sufrimiento de los hombres, por la desproporción, arbitrariedad y discriminación veleidosa con la que impone la pena? ¿No sería legítima, por mor del mismo principio de retribución que justifica el castigo por los pecados, la queja del justo sufriente? Así llega a creerlo Job cuando interpela a Dios, cuando eleva a Dios y a sus hipócritas valedores (Elifaz, Bildad y Sofar) su inflamado alegato. ${ }^{17}$

De las experiencias dolorosas y sufrientes emergen cuestiones a las cuales intentamos dar respuesta, de este modo entra en juego la función donadora de sentido al mundo y a la vida. Esta función poiética construye tales concepciones

16 Nicol 1950, p. 20

17 Fernández Liria 2005, p. 172 
del mundo y de la vida. De estas cuestiones que emergen de las experiencias algopháticas podemos nombrar las siguientes: ¿Por qué ha ocurrido? ¿Se podría haber evitado? ¿Quién o quiénes son los responsables? ¿Es justo? Todas estas cuestiones se formulan bajo el principio de retribución, que es común a muchas otras religiones, y que se puede englobar bajo la denominada lógica kármica, siguiendo la estela de las religiones orientales como son el budismo, el hinduismo o el jainismo. ${ }^{18}$ Junto a estas cuestiones también hay preguntas que afectan directamente al modo de existencia y que también son ético-políticas como la siguiente: ¿cómo he de vivir?

Por eso, más allá de la propias vivencias de Job y de cómo éste las interpreta, lo que nos encontramos en la tradición cristiana es con un mártir (Santo Job), que se transfigura en modelo de existencia mediante el cual se traduce el dolor y sufrimiento de nuestras vidas en prueba existencial. En la tradición bíblica, el sufrimiento y el dolor aparecen después de que Adán y Eva hayan cometido el pecado original.

La muerte de Jesucristo en la cruz es en esencia un misterio del sufrimiento, un relato de la redención por un dolor infinito, el único adecuado para absorber el infinito pecado de los seres humanos. Durante largo tiempo, para el cristiano el dolor es participación en una modalidad menor de los ejemplares sufrimientos de Cristo, cuya intensidad sabe que nunca conseguirá alcanzar. ${ }^{19}$

Sin embargo, no es casualidad que el conocido como «primer argumento» ateísta proceda de la apelación al Dios omnisciente, omnipotente y eternamente bondadoso ante su creación doliente y sufriente de guerras, hambrunas y otros acontecimientos interpretados como injustos. Esta apelación a la diké teológica, es decir, a la justicia divina, es conocida como paradoja de Epicuro. Para solventar dicha paradoja, se apela a las diferencias entre la razón divina y humana: hay razones de Dios que el humano nunca podrá llegar a entender. Este dolor y sufrimiento entra a formar parte de una teodicea.

Con este ejemplo se muestra el carácter poiético de la donación de sentido al mundo y a la vida, pero también se muestra el carácter autopiético en tanto que la donación de sentido no es solo sobre el sentido externo, sino que apela al modo en cómo debemos vivir. La concepción del mundo y de la vida es performativa en tanto en cuanto modifica nuestra conducta, nuestro modo de ser y estar en el mundo.

18 Cfr. Le Breton 1999, p. 119-123

19 Le Breton 1999, p. 108 


\section{CONCEPCIONES DEL MUNDO Y DE LA VIDA.}

EN TORNO AL DOLOR Y EL SUFRIMIENTO PRODUCIDO

En el apartado anterior nos hemos centrado en las concepciones del mundo y de la vida en torno al dolor y el sufrimiento padecido. Se ha mostrado cómo las experiencias algopháticas no solo tienen una dimensión subjetiva sino que estas vivencias son interpretadas desde concepciones del mundo y de la vida que otorgan sentido a dichas vivencias. Esta dimensión es intersubjetiva en tanto en cuanto hacen referencia al sentido socialmente compartido.

La intersubjetividad del dolor y el sufrimiento tiene que ver con las relaciones con los otros y, dentro de esta dimensión intersubjetiva, se ha hecho referencia a la relación simbólica, de ahí la apelación a las concepciones del mundo y de la vida. Sin embargo, en esta dimensión intersubjetiva no todo dolor y sufrimiento es paciente, también existe el dolor y el sufrimiento producido. Cabe distinguir, por tanto, entre el dolor paciente (el que se padece) y el dolor agente (el que se produce). El dolor paciente, cuando se expresa, se manifiesta en formas de llanto, queja, gritos, etc. Son expresiones que reclaman atención y acompañamiento del Otro. Mientras que el dolor agente es el dolor infligido en y sobre el Otro.

Las expresiones de dolor y sufrimiento padecido son relaciones intersubjetivas de las cuales se puede decir que son mecanismos que establecen lazos comunitarios. Sin embargo, es la agencia del dolor y el sufrimiento el elemento de ordenación de la vida social. Del dolor agente se puede decir - en terminología foucaultiana - que constituye la gobernabilidad de la comunidad en tanto en cuanto se instituye como tecnología social. ${ }^{20}$ Los mecanismos de generación de ordenación de la vida social son varios: de integración, de corrección, de liberación, de control, etc. En este sentido nos dirá Le Breton que:

El dolor infligido al individuo desprotegido es un medio de gobierno del otro, de dominio sobre su comportamiento, sino sobre su conciencia [...] Toda transgresión del código moral implica, por parte de los representantes de la ley, el reparto de un dolor dosificado, en principio, de acuerdo con la importancia de la falta cometida. El dolor administrado es castigo, marca en la carne el defecto moral o que se presume como tal; sanciona la conducta errónea. ${ }^{21}$

Los mecanismos de ordenación de la vida social que se apoyan en el dolor y el sufrimiento agente se develan de una manera más nítida cuando nos remitimos a términos como el de disciplina. El término disciplina puede

20 Para el sentido de tecnología y gobernabilidad en Foucault consultar: Foucault 1990, pp. $48-49$

21 Le Breton 1999, p. 234 
hacer alusión tanto a una doctrina, como al comportamiento militar, así como un código moral de conductas y reglas. Sin embargo, este término también ha adquirido otro significado y es el de castigo, sobre todo a partir del latín eclesiástico de la Edad Media. ${ }^{22}$ La disciplina, en tanto que castigo, se convierte en una tecnología social a través de lo que es el dolor agente. Por este motivo, no cabe sorprenderse de los nexos etimológicos de ciertas palabras: en griego y latín poine significa sufrir, mientras que poena significa castigo. Existe, por tanto, un nexo con dolor en idiomas tales como el inglés pain como en alemán pein. Si bien el castigo como ejemplo de mecanismo de la ordenación de la vida social puede servirnos para cierta cultura occidental, el dolor agente como regulador de la vida en sociedad se puede observar en otras culturas.

En la etnología se han estudiado diferentes procesos conocidos como rituales de iniciación. Se trata de mecanismos sociales que dividen en fronteras simbólicas los grupos sociales. En ciertas culturas los ritos de iniciación tienen que ver con el tránsito de la vida del infante a la vida adulta. En ocasiones estos ritos suponen pruebas que podemos tildar de dolorosas, como es el caso del paso a la vida adulta y el reconocimiento de estatus social en la población bukusu, en Kenia. La sociedad bukusu lleva cabo la práctica de la circuncisión para simbolizar el tránsito de la vida infante a la vida adulta, entre otras cosas. En este ritual, el niño que va a ser mutilado no tiene que mostrar ningún tipo de expresión de miedo y, cuando están cortando el prepucio, el niño no tiene que mostrar signos de dolor. ${ }^{23}$ Otro ejemplo de ritual donde el dolor agente supone un mecanismo que simboliza un tránsito social es el de la población Sateré-Mawé, que reside en el río Amazonas de Brasil. En esta población se lleva a cabo una prueba que mide la «casabilidad» (posibilidad o no de casarse) que consiste en ponerse un guante repleto de hormigas bala. ${ }^{24}$ Más allá de los casos particulares que aquí se exponen a modo ejemplo, vemos cómo el dolor agente cumple una función social que estructura y organiza la sociedad. De este modo el dolor agente se convierte en una biotecnología que adquiere sentido dentro de una concepción del mundo y de la vida concreta situada en un espacio-tiempo. De este modo Antonio Madrid dice que:

El sufrimiento posee un carácter fundante en la historia política de los pueblos. Cada régimen político reserva un lugar privilegiado para sus héroes, salvadores o mártires — también para sus enemigos más encarnizados-. Desde las primeras narraciones políticas ha sido así. El sufrimiento que cuenta, el que adquiere carácter fundante, es aquél con el que se identifican los miembros de la comunidad o bien aquel que un grupo dominante propone, difunde e impone como referente

$22 C f$. Le Breton1999, p. 235

23 Para saber más acerca de este ritual puede consultarse: Mbachi y Likoko 2013

24 Para saber más acerca de este ritual puede consultarse: Alvarez 2011 
político. Se establece de esta forma una relación de distinción, identificación y validación, que actúa como relación de solidaridad política entre el sufrimiento fundante y la situación vital de la comunidad. ${ }^{25}$

Si bien el dolor es una experiencia es privativa en tanto en cuanto el doliente tiene vivencia de su dolor en primera persona, el padecimiento del mismo es interpretado de maneras distintas. Desde una concepción teológica cristiana la experiencia algopáthica es interpretada como una teodicea; desde una concepción mitológica como la de los bukusu, el dolor se interpreta como prueba del tránsito a una vida adulta; o desde la concepción de los Sateré-Mawé el dolor es prueba de la casabilidad. Lo que tienen en común todas estas concepciones es que estructuran y ordenan la vida en sociedad en base una concepción del mundo y de la vida.

\section{CONSECUENCIAS FILOSÓFICAS}

Si el dolor fuera una mera reacción bioquímica que respondiera a un proceso fisiológico, no se podría entender la pluralidad de sentidos que adquieren las experiencias algopháticas así como tampoco se entendería la pluralidad de mecanismos biopolíticos de socialización que regulan y ordenan la vida en sociedad. Esta propuesta de comprensión del dolor y el sufrimiento desde las concepciones del mundo y de la vida deviene de las investigaciones que han introducido variables culturales en los estudios acerca del dolor. Sin embargo, no se parte de la premisa de las concepciones del mundo y de la vida, sino que son consecuencia de la pluralidad de sentidos que articulan de manera fáctica la organización social.

En esta comprensión e interpretación, por tanto, no se parte de la evaluación y validez de cada particular Weltanschauung, sino que se toma la facticidad de las mismas para comprender al ser humano. De este modo, el ser humano no puede definirse por una de sus plurales actividades como se había hecho tradicionalmente al definir la esencia de lo humano a través de categorías tales como homo sapiens, homo faber, homo ludens, etc. Como vemos, la poiesis humana es donadora y generadora de sentido pero, no solo el sentido articula la concepción del mundo y de la vida sino que además tiene carácter autopoiético en tanto que nos transforma a nosotros mismos en nuestro modo de existir. Es así como se puede argumentar que cuando se afirma que el dolor es un mecanismo de supervivencia se está cayendo en un reduccionismo en tanto en cuanto solo se está pensando desde el dolor en sentido somático y funcional. De este punto de vista se puede entender la función de la farmacología donde el dolor y el sufrimiento es síntoma a aliviar o erradicar. Pero el dolor y el 
sufrimiento no solo es experiencia subjetiva privativa sino que es experiencia intersubjetiva articulada desde la esfera de lo simbólico.

Pero la compresión e interpretación del dolor y el sufrimiento desde su dimensión simbólica no solo tiene consecuencias teóricas respecto a la concepción antropológica, sino que también tiene consecuencias prácticas. Por ejemplo, si el dolor tuviera una función única de supervivencia, ¿qué sentido tiene el dolor del parto? O, dado que el sentido es poiesis mundana, ¿qué sentido tiene seguir sufriendo cuando se tiene una enfermedad terminal?

Reconociendo que existen prácticas de gestión del dolor y el sufrimiento y que estas dependen de las concepciones del mundo y de la vida, vemos cómo estas son contingentes. Es decir, se dan de tal forma pero podrían haber sido de otra. Cuando se plantea la cuestión de la violencia obstetricia tiene que ver precisamente con el cuestionamiento de las técnicas utilizadas para la asistencia al parto. Respecto a cómo afrontar el sufrimiento desde una enfermedad terminal se puede argumentar que dado que existe una pluralidad de concepciones del mundo y de la vida, desde el reconocimiento de la persona como sujeto inserto en un horizonte de sentido determinado, ¿por qué no puede ser dueño de su destino el enfermo terminal? Ya sea poner fin a su existencia, ya sea aceptar el dolor y el sufrimiento.

Estas cuestiones quedan abiertas, dado que el objeto de este artículo es tan solo mostrar el carácter simbólico del dolor y el sufrimiento. Se inserta dentro de la crítica a los reduccionismos que son tales en cuanto exceden su campo de investigación. La filosofía entendida como saber y actividad de segundo grado con intención holótica implica replantearse la concepción antropológica $\mathrm{y}$, en este caso, se considera a los seres humanos como seres simbólicos donde las concepciones del mundo y de la vida suponen una brújula y mapa vital de ordenación de sentido y donde el conjunto de prácticas sociales junto a las técnicas y tecnologías de los respectivos presentes articulan la ordenación de la esfera social.

\section{REFERENCIAS BIBLIOGRÁFICAS}

ALVAREZ, G. O. (2011): «Pós-dadiviano Sateré-Mawé: parentesco y rituales de afibiliadad», Vibrant: Virtual Brazilian Antrophology, Vol. 8, No. 2, juliodiciembre, pp. 373-402

BUENO, G. (1995): ¿Qué es la ciencia? La respuesta de la teoría del cierre categorial. Ciencia y Filosofía. Oviedo: Pentalfa

DILTHEY, W. (1944): Introducción a las ciencias del espíritu. Obras completas: Tomo I. Eugenio Imaz (trad.). México: Fondo de Cultura Económica

Madrid 2010, p. 76 
DILTHEY, W. (1945): Teoría de la concepción del mundo. Obras completas: Tomo VIII. Eugenio Imaz (trad.). México: Fondo de Cultura Económica

, (1978): Teoría de la concepción del mundo. Obras completas: Tomo VIII.

Eugenio Imaz (trad.). México: Fondo de Cultura Económica

, (1988): Teoría de la concepción del mundo. Julián Marías (trad.). Madrid:

Alianza Editorial

FERNÁNDEZ LIRIA, P. (2005): «Reflexiones sobre Job. En torno al problema del mal, el sufrimiento del justo y la Teodicea», Logos. Anales del Seminario de Metafísica, Vol. 38

FOUCAULT, M. (1990): Tecnologías del yo. Mercedes Allendesalazar (trad.). Barcelona: Paidós

GEERTZ, C. (2003): La interpretación de las culturas. Alberto L. Bixio (trad.). Barcelona: Gedisa

HUSSERL, E. (2008): La crisis de las ciencias europeas y la fenomenología trascendental. Julia V. Iribarne (trad.). Buenos Aires: Prometeo Libros

LE BRETON, D (1999): Antropología del dolor. Daniel Alcoba (trad.). Barcelona: Editorial Seix Baral

MADRID, A. (2010): La política y la justicia del sufrimiento. Madrid: Trotta

MBACHI, F. Y LIKOKO, S. (2013): «Traditional male circumcision and its implications on the socio-economic and psychosocial life of the bukusu community in Kenya», Research on humanities and social sciences, Vol. 3, No. 1, pp. 218-224

NICOL, E. (1950): Historicismo y existencialismo. La temporalidad del ser y la razón. México: El Colegio de México

NICOL, E. (2013): La idea del hombre. México: Fondo de Cultura Económica

PARÍS, C. (2014): Ética radical. Los abismos de la actual civilización. Madrid: Tecnos

SERRANO DE HARO, A. (2012): «A propósito de la fenomenología del dolor», Crítica, No. 981, septiembre-octubre, pp. 12-16

Francisco Javier Cortés SÁnchez es doctor en Filosofía por la Universidad de Salamanca con mención Internacional. Realizó su doctorado gracias a la ayuda de contratación predoctoral de Personal Investigador de la Junta de CyL cofinanciado por el Fondo Social Europeo. Ha realizado estancias de investigación en la UNAM y en el IFS del CSIC.

Actualmente es Profesor Asociado de la Universidad de Salamanca.

Lineas de investigación

Hermenéutica, fenomenología, metafísica y ontología, filosofía contemporánea, filosofía del exilio español, Dilthey y Eduardo Nicol 
Publicaciones recientes

(2015): «Dilthey, de la mundaneidad a la metafísica», Euphyía. Revista de Filosofía, Año 9-16, enero-julio, pp. 9-30

(2014): «La fenomenología dialéctica de Eduardo Nicol», Investigaciones fenomenológicas, No 11, pp. 57-79

Email:

francortes@usal.es 
